\title{
Sub-Milliarcsecond Binary Star Astrometry ${ }^{1}$
}

\author{
XIAOPEI PAN \\ California Institute of Technology, Pasadena, CA 91125, USA \\ MICHAEL SHAO \& M. MARK COLAVITA \\ Jet Propulsion Laboratory, Pasadena, CA 91109, USA
}

\begin{abstract}
Long-baseline optical interferometry has revolutionised binary star astronomy. This paper reviews some recent binary star work done using the Mark III Stellar Interferometer. For the first time, many spectroscopic binaries, eclipsing binaries, triple systems, and binaries with X-ray sources have had their visual orbit, diameter of the primary component, and color index determined. Also, accurate stellar masses, distances, radii, and effective temperatures have been derived. A preliminary distance to the Hyades cluster has also been determined. In addition, preliminary observations provide evidence of a third body in the binary star system $\zeta$ Her, which may resolve an 85-year old discrepancy in astronomy.
\end{abstract}

\section{INTRODUCTION}

Binary stars play an important role in astronomy, providing direct determination of fundamental physical characteristics of a star, such as mass, radius, temperature, composition, and distance. Binary and multiple stars are very common; single stars are the minority in the stellar population. Traditionally, binary stars are classified as visual or spectroscopic, depending on their separation. Originally, the breakpoint between the classes was set by the resolution of a large telescope, limited by atmospheric turbulence to $0 " .5-1 "$. In the past two decades, speckle interferometry has pushed the angular resolution to the diffraction limit, $\sim 33$ mas for a 4-m telescope. However, a large gap between the spectroscopic and visual binaries still remains. Less than 150 stars on the Hertzsprung-Russell diagram have had masses determined to a precision of $5 \%$ or better so far; the measurement precision of stellar luminosity for close binaries is even worse.

The Mark III Stellar Interferometer (Shao et al. 1988), a long-baseline optical interferometer in routine operation since 1988, demonstrates milliarc second resolution, sub-milliarcsecond (mas) precision, and detectability of a 4mag difference between components. With this instrument, many close binary stars have been resolved, and all of the orbital elements of a binary star can be determined independently, including three elements, angular semimajor axis $a^{\prime \prime}$, inclination $i$, and position angle of the ascending nodes $\Omega$, which cannot be determined spectroscopically (Pan et al. 1990a). The precision of the individual data points in the orbit has reached 0.2 mas. For many binary stars, not only the binary system itself has been resolved, but also the diameter of the primary component has been determined. In addition, the magnitude differences between

\footnotetext{
${ }^{1}$ This contributed paper was judged outstanding by the Colloquium participants and is therefore included in the proceeding with the invited papers - eds
} 
the components at different wavelengths have been determined to a precision of $0.1 \mathrm{mag}$.

At present, more than 25 spectroscopic binary stars have been resolved; the closest separation of the two components is only 3 mas, the smallest semimajor axis is 6 mas, the largest magnitude difference is $4 \mathrm{mag}$, the most distant system is $320 \mathrm{pc}$, and the diameters of the primary range from less than 1 mas to 8.2 mas. Their spectra are distributed from B3 to G8 types. Because of the limit on paper size, only a brief description of the results on binary stars with the Mark III Stellar Interferometer will be presented here.

\section{OBSERVATIONS WITH THE MARK III STELLAR INTERFEROMETER}

For binary star observations, a variable baseline is used with the Mark III interferometer, which has two siderostats separated by 3 to $32 \mathrm{~m}$ oriented northsouth. Fringes are observed simultaneously in four spectral channels: a wideband channel at $700 \mathrm{~nm}$ for fringe tracking, and three narrow-band channels for the visibility measurements at $800 \mathrm{~nm}, 550 \mathrm{~nm}$, and $500 \mathrm{~nm}$, respectively. Each observation has fringe tracking data recorded over a $75 \mathrm{~s}$ interval, followed by a $5 \mathrm{~s}$ measurement of the total dark count and sky background. The instrument is highly automated. Typically, 160 to 200 observations can be made each night, and 15 to 25 observations per night are planned for each binary star.

The squared fringe visibility, $V^{2}(u, v)$, is given (Pan et al. 1992) by

$$
V^{2}(u, v)=\left\{\Gamma^{2}\left(\theta_{1}\right)+\left[\gamma R \Gamma\left(\theta_{2}\right)\right]^{2}+2 \gamma R \Gamma\left(\theta_{1}\right) \Gamma\left(\theta_{2}\right) \cos \phi\right\} /(1+R)^{2},
$$

where

$$
\begin{aligned}
\Gamma\left(\theta_{i}\right) & =2 J_{1}\left(\pi b \theta_{i} / \lambda\right) /\left(\pi b \theta_{i} / \lambda\right) \quad(i=1,2) \\
\gamma & =\sin [\phi(\Delta \lambda / \lambda) / 2] /[\phi(\Delta \lambda / \lambda) / 2] \\
\phi & =2 \pi\left(U S_{R . A .}+V S_{D e c}\right) / \lambda
\end{aligned}
$$

In these expressions, $R$ is an intensity ratio, $J_{1}(x)$ is a first-order Bessel function, $\lambda$ is the wavelength, $b$ is the length of projected baseline, $\theta_{i}$ are the angular diameters of each component, $\Delta \lambda$ is the bandwidth of a filter with a rectangular bandpass, $U$ and $V$ are the components of the projected baseline on the $u-v$ plane, and $S_{R . A}$ and $S_{D e d}$ are the angular separations between the primary and the companion in right ascension and declination, respectively. As a function of time, the measured visibilities will have periodic changes depending on the baseline, the wavelength, the relative positions and brightnesses of two components, as well as their angular diameters.

Typical model fitting of the squared fringe visibilities as a function of time are shown in Figures $1-4$. These plots indicate that for large separations, long baselines, and short wavelengths, the periodic changes occur rapidly. Also, for larger differences of the brightness, the smaller amplitude of the changes makes detection more difficult. The peak value of the fringe visibility curves mainly depends on the diameter of the primary component. It is clear that this automated instrument provides the possibility of many observations per 


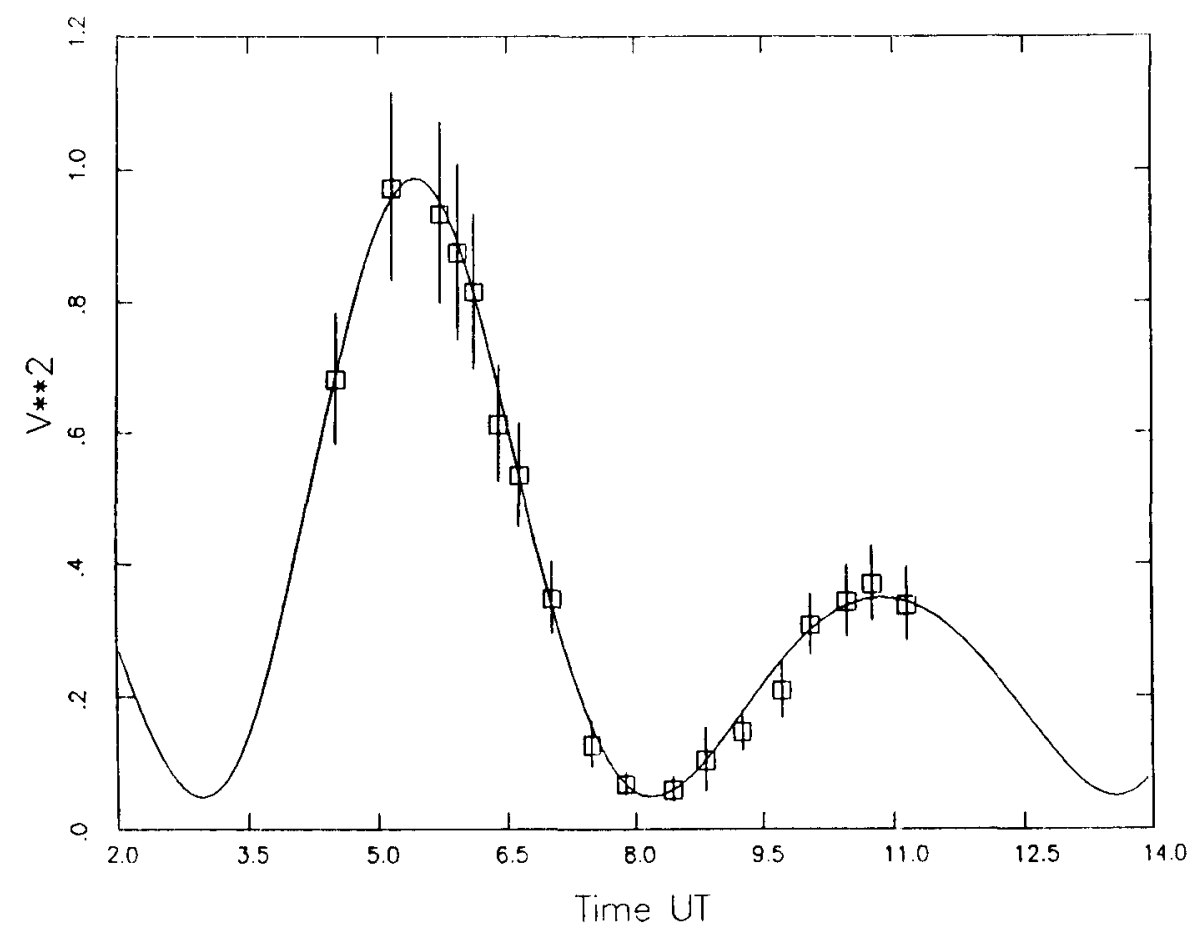

FIGURE 1. Measured and best-fit fringe visibilities for $\beta$ Tri at $550 \mathrm{~nm}$ on $28 \mathrm{~m}$ baseline, Nov. 5, 1989. The magnitude difference is 0.5 ; separations in R.A. and Decl. are 5.0 mas and -5.4 mas, respectively.

star per night, and ensures good model fitting for accurate determination of the parameters of binary stars.

Angular resolution with the Mark III interferometer on the longest baseline reaches 3 mas at $500 \mathrm{~nm}, 4$ mas at $550 \mathrm{~nm}$, and 6 mas at $800 \mathrm{~nm}$. Close binary stars have been resolved with rms dispersions of the observations from the orbit model to about 0.2 mas.

A good example is provided by interferometric observations of the well known spectroscopic binary $\beta$ Ari (HR 553), which has a very large orbital eccentricity $(e \sim 0.9)$. While this binary was predicted to be resolvable for a long time, the system has been resolved only by the Mark III. All of the orbital parameters of this binary star have been determined independently of spectroscopic data (Pan et al. 1990b). In fact, three orbital parameters can only be obtained from interferometric data, i.e. , $a^{\prime \prime}=0^{\prime \prime} .03654 \pm 0^{\prime \prime} .00023, i=45^{\circ} .94$ $\pm 0^{\circ} .57$, and $\Omega=77^{\circ} .54 \pm 0^{\circ} .80$. The other orbital parameters for this system show good agreement between interferometric and spectroscopic results.

\section{RESOLUTION AND PRECISION}

Recent data with the Mark III provides evidence for a third body in the binary $\operatorname{star} \zeta$ Her (HR 6212). This visual binary $\operatorname{star}\left(V=2^{m} \cdot 80, \Delta V=2^{m} .63, \mathrm{G} 0\right.$ 


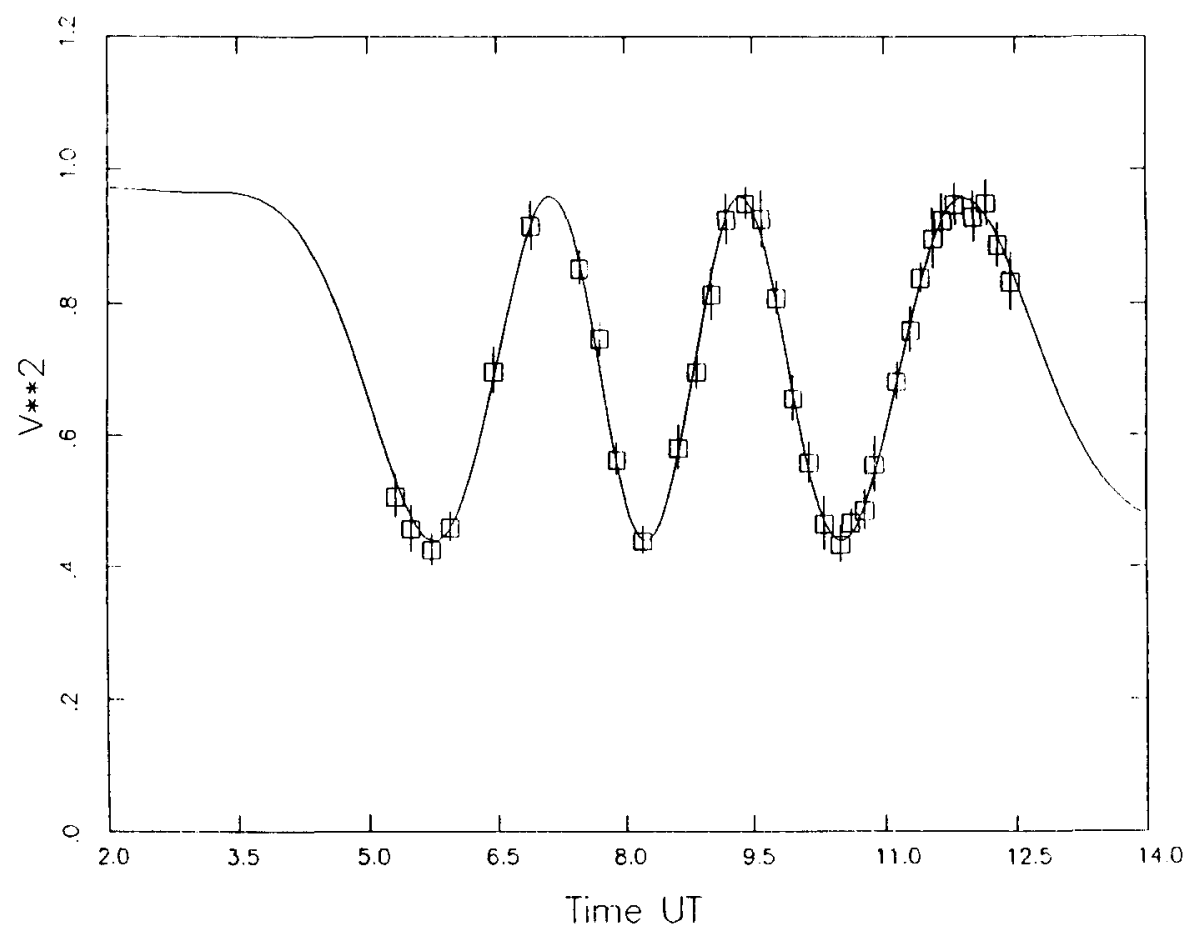

FIGURE 2. Measured and best-fit fringe visibilities for $\alpha$ And at $800 \mathrm{~nm}$ on $32 \mathrm{~m}$ baseline, Aug. 31, 1989. The magnitude difference is 1.9; separations in R.A. and Decl. are $-15.9 \mathrm{mas}$ and $5.2 \mathrm{mas}$, respectively.

IV) was discovered in 1782 . Several orbital solutions have been published since 1906 , and from time to time a third body has been suggested for this system. The visual pair has a period of $34^{y} .487$ and semimajor axis of $\sim 1^{\prime \prime} .3$. This system has also been observed by speckle interferometry. Unfortunately, disagreement between the spectroscopic and visual observations has blocked any possibility of finding a satisfactory orbit. This system has also attracted the attention of astrometric observers. The latest astrometric study in 1981 determined a parallax $\pi^{\prime \prime}=0^{\prime \prime} .098 \pm 0^{\prime \prime} .005$ and mass fraction $B=0.357 \pm 0.023$. However, these results produced significant differences for the masses and absolute magnitudes of the components of this system as determined from spectroscopic and visual observations. New observations with the Mark III interferometer provide evidence for a third body in this system, with a separation of order 10 mas, a magnitude difference of $\sim 3.5$ mag between the two components of the primary, and a diameter of $\sim 2.2$ mas for the primary of the interferometric pair. We hope that the discrepancy among spectroscopic, visual, astrometric, and speckle interferometric results will soon be solved. 


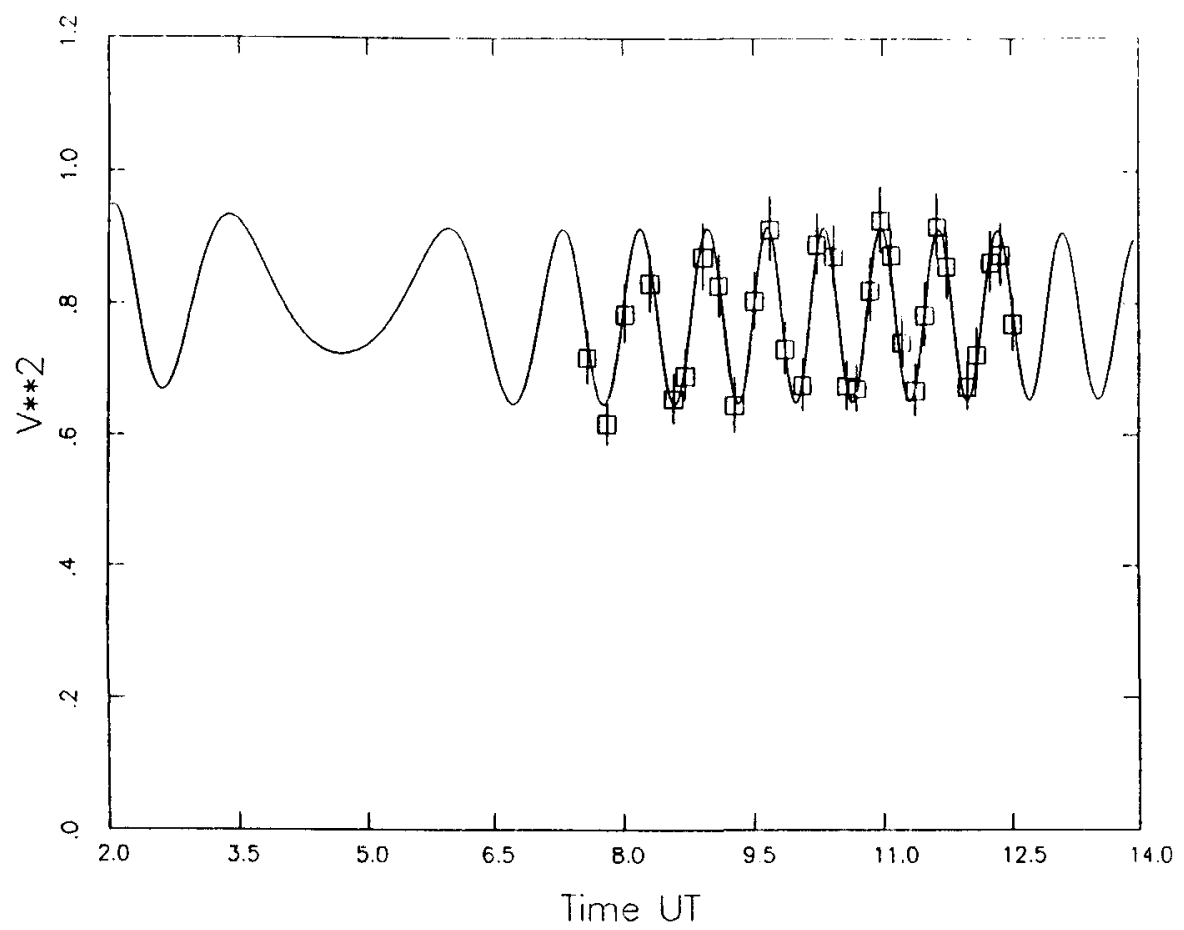

FIGURE 3. Measured and best-fit fringe visibilities for $\beta$ Ari at $800 \mathrm{~nm}$ on $32 \mathrm{~m}$ baseline, Aug. 31, 1989. The magnitude difference is 2.6; separations in R.A. and Decl. are $\mathbf{5 4 . 1}$ mas and $\mathbf{- 1 7 . 0}$ mas, respectively.

\section{MAGNITUDE DIFFERENCES AND COLOR INDEX}

Luminosity is the one of the fundamental physical parameters of a star. Longbaseline interferometry can provide an accurate measurement of magnitude difference.

For example, the visual orbit of the spectroscopic binary $\alpha$ And has been determined with the Mark III interferometer (Pan et al. 1992), and agrees well with the spectroscopic results. Particularly, the magnitude differences are measured as $1^{m} .82 \pm 0^{m} .04$ at $800 \mathrm{~nm}$ and $1^{m} .99 \pm 0.04$ at $550 \mathrm{~nm}$. Given a total visual magnitude $m_{v}=2^{m} .06$, the visual magnitudes of the primary and the companion are determined as $2^{m} .22 \pm 0^{m} .01$ and $4^{m} .21 \pm 0^{m} .04$, respectively. Utilizing the multi-color photometric observations $V-R=-0^{m} .03$ and $V-I$ $=-0^{m} .13, V-800 \mathrm{~nm}$ is estimated as $-0^{m} .08 \pm 0^{m} .02$, and the magnitude of the two components of $\alpha$ And at $800 \mathrm{~nm}$ are calculated as $2^{m} .33 \pm 0^{m} .02$ and $4^{m} .15$ $\pm 0^{m} .04$, respectively. Thus, the color indices of the primary and the companion between 550 and $800 \mathrm{~nm}$ are determined as $-0^{m} .11 \pm 0^{m} .03$ and $+0^{m} .07 \pm$ $0^{m} .05$. These results indicate that the blue primary and the white companion correspond to a late-type B star and an early-type A star. The result for the primary is consistent with the classification of stellar spectra. 


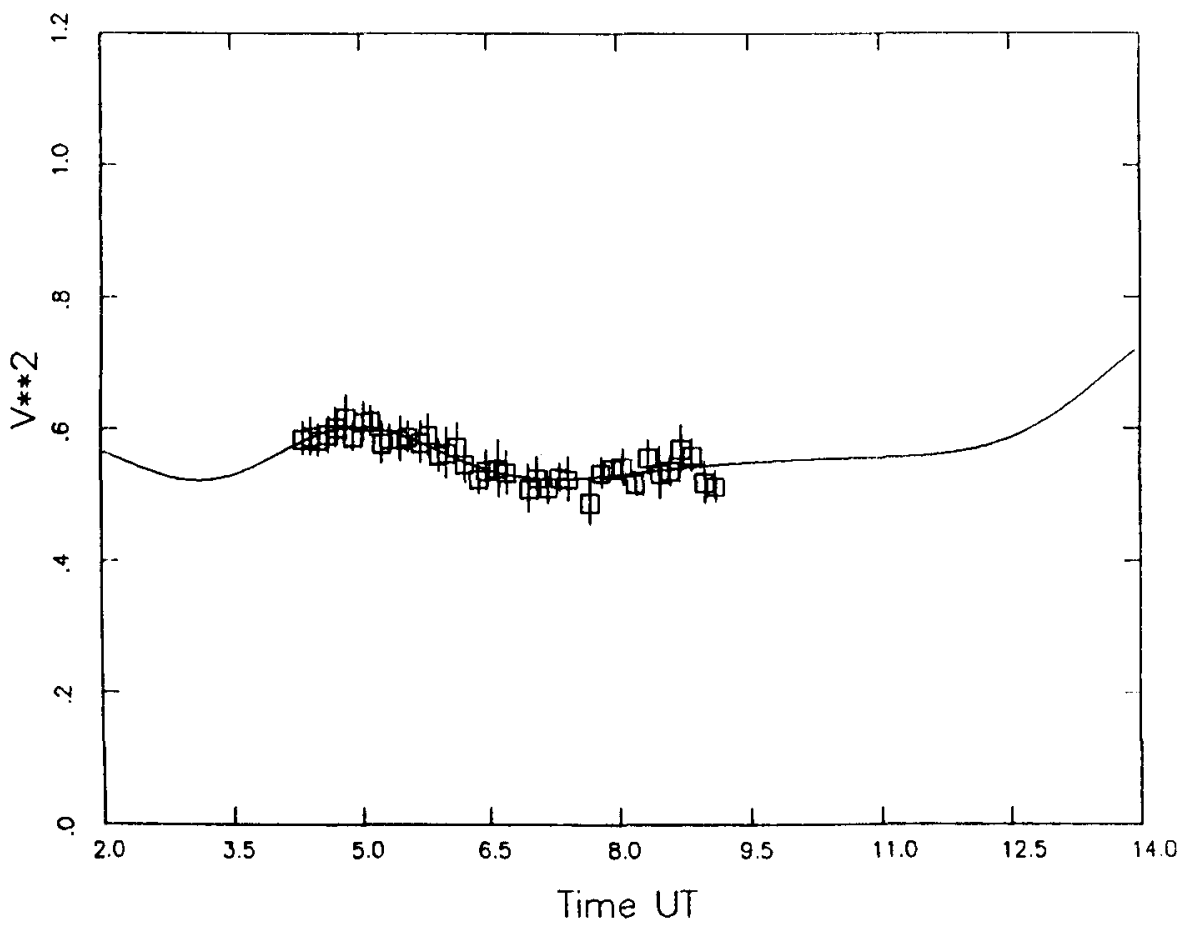

FIGURE 4. Measured and best-fit fringe visibilities for $\beta$ Her at $800 \mathrm{~nm}$ on $28 \mathrm{~m}$ baseline, Jul. 14, 1990. The magnitude difference is 4.0; separations in R.A. and Decl. are -13.4 mas and $11.2 \mathrm{mas}$, respectively. The diameter of the primary is 3.2 mas.

\section{DISTANCES AND MASSES}

Binary stars provide the only method for the direct determination of stellar masses, and provide an opportunity to measure astronomical distances with much better precision for more distant objects than is possible with trigonometric parallax techniques.

The brightest visual binary star in the Hyades cluster, $\theta^{2}$ Tau (HR 1412) has been resolved with the Mark III interferometer (Shao et al. 1991). The preliminary results are $a^{\prime \prime}=0^{\prime \prime} .0186 \pm 0^{\prime \prime} .0002, i=46^{\circ} .2 \pm 1^{\circ} .0, \Omega=171^{\circ} .2 \pm 1^{\circ} .8$, and $\omega=57^{\circ} .7 \pm 1^{\circ} .7$. The magnitude differences between the two components are $\Delta m=1^{m} .15 \pm 0^{m} .07$ at $800 \mathrm{~nm}, \Delta m=1^{m} .18 \pm 0^{m} .10$ at $550 \mathrm{~nm}$, and $\Delta m=$ $1^{m} .16 \pm 0^{m} .13$ at $450 \mathrm{~nm}$, yielding individual visual magnitudes of $m_{v 1}=3^{m} .72$ and $m_{v 2}=4^{m} .91$ at $550 \mathrm{~nm}$. Using the mass ratio and the linear semimajor axis of the primary from spectroscopy, a preliminary estimate of the distance to the Hyades cluster $d=43.7 \pm 1.9 \mathrm{pc}$, and individual masses $\mathcal{M}_{1}=2.0 \mathcal{M}_{\odot}$ and $\mathcal{M}_{2}=1.6 \mathcal{M}_{\odot}$ are derived. The accuracy of the distance measurement to the Hyades cluster could reach $1 \mathrm{pc}$ or less if the spectroscopic measurements can be improved. Both components of $\theta^{2}$ Tau agree well with the empirical massluminosity diagram. 


\section{STELLAR DIAMETER AND EFFECTIVE TEMPERATURE}

The binary star $\beta$ Her (HR 6148) was first detected as a spectroscopic binary in 1899 , and had its orbit determined in 1908 as follows: $P=410^{d} .575, T=\mathrm{JD}$ $2415500.374, e=0.5498$, and $\omega=24^{\circ} .6$. While this system has been observed using speckle interferometry a number of times from $1975-1986$, the results for $\beta$ Her do not produce a reasonable visual orbit.

The visual orbit, the stellar diameter of the primary component, and the magnitude difference of the spectroscopic binary $\beta$ Her have recently been determined directly with the Mark III Stellar Interferometer (Pan et al. 1991). Observations in 1989 were analyzed to determine the orbital elements of this system, and predictions of its orbital motion for 1990 and 1991 agree well with the observational results. The orbital elements of $\beta$ Her are determined as follows: $a^{\prime \prime}=0^{\prime \prime} .0621 \pm 0^{\prime \prime} .0002, i=100^{\circ} .2 \pm 0^{\circ} .1, \Omega=9^{\circ} .2 \pm 0^{\circ} .4$, and $\omega=103^{\circ} .6 \pm$ $0^{\circ} .1$. The stellar diameter is determined as $\theta^{\prime \prime}=0^{\prime \prime} .0032 \pm 0^{\prime \prime} .0001$. The magnitude differences between the two components are $\Delta m=3^{m} .9$ at $800 \mathrm{~nm}$ and $\Delta m$ $=3^{m} .7$ at $550 \mathrm{~nm}$, yielding individual visual magnitudes of $m_{v 1}=2^{m} .8$ and $m_{v 2}$ $=6^{m} .5$, respectively. From the estimated mass ratio, masses of the primary $\mathcal{M}_{1}$ $=2.9 \mathcal{M}_{\odot}$ and the companion $\mathcal{M}_{2}=0.9 \mathcal{M}_{\odot}$ are derived. The linear radius and the effective temperature of the primary are $R_{1}=9.3 \mathrm{R}_{\odot}$ and $T_{\text {eff } 1}=5332^{\circ} \mathrm{K}$, respectively. This is a precise indicator of a G8 spectral type for this giant star.

\section{CONCLUSION}

Long-baseline optical interferometry has proved to be a powerful and productive technique for binary star astronomy. We expect that more close binary stars will be resolved in the near future.

\section{REFERENCES}

Pan, X.P. et al. 1990a, SPIE, 1237, 301

Pan, X.P., Shao, M., Colavita, M.M., Mozurkewich, D., Simon, R.S., \& Johnston, K.J. 1990b, ApJ, 356, 641

Pan, X.P. et al. 1991, BAAS, 22, 1335

Pan, X.P., Shao, M., Colavita, M.M., Armstrong, J.T., Mozurkewich, D., Vivekand, M., Denison, C.S., Simon, R.S., \& Johnston, K.J. 1992, ApJ, 384, 624

Shao, M., Colavita, M.M., Hines, B.E., Staelin, D.H., Hutter, D.J., Johnston, K.J., Mosurkewich, D., Simon, R.S., Hershey, J.L., Hughes, J.A., \& Kaplan, G.H. 1988, $A \& A, 193,357$

Shao, M. et al. 1991, BAAS, 23, 830

\section{DISCUSSION}

MOURARD: What kind of disk model is used when fitting the visibility data, especially in the case of resolved components?

PAN: At present, we use a uniform disk model. 
ZINNECKER: Are there any eclipsing binaries which you can resolve and for which you can even determine the stellar diameters?

PAN: Yes, I have observed several eclipsing binaries. In particular, we have obtained very interesting results for Algol and will publish them soon.

SCARFE: I have followed the radial velocity $\zeta$ Her A for nearly 30 years. They fit a 2-body orbit with an rms scatter of $0.25 \mathrm{~km} \mathrm{~s}^{-1}$. I think this puts severe constraints on the inclination of the orbit of your newly-discovered component.

PAN: I will make frequent observations of this system so that we will hopefully have some conclusion soon.

GRIFFIN: Colin Scarfe's radial velocities were so good that I promptly dropped $\zeta$ Her from my own observing program as if it were a hot brick!

FEKEL: Most of the systems you discussed appear to be late-type stars. Are there any restrictions on observing early-type stars?

PAN: No, in fact, one of our best determined systems is $\alpha$ And with a spectral type of B8 IV. For the binaries we've resolved so far, their spectral types range from B3 to G8.

ARMSTRONG Several other systems observed with the Mark III are also early type systems, such as $\pi$ And, so there is no real restriction.

VAN DESSEL: About $\beta$ Arietis; it has a high eccentricity orbit and could well be prone to mass loss at periastron passage and change of orbital elements. Have you any comments on your data compared to previous results?

PAN: This is a very important question which I have had in mind for a long time. I am planning to obtain more data this year to look try to make some conclusions about this from our measurements.

McALISTER: Do you know where $\theta^{2}$ Tauri is located with respect to the cluster; i.e. because there is considerable dispersion in the distance to individual cluster members, how close is $\theta^{2}$ Tauri to the mean cluster distance?

PAN: I will check on this.

MCALISTER: What is the future of the Mark III?

ARMSTRONG: We have been extending Mark III operations year by year. Right now, we think we will observe at least until we get fringes with our new interferometers at Lowell. But we do not have the manpower to fix a major problem; a failure of the wire-wrap electronics would be the biggest problem. 